\title{
Improving Access to a Digital Video Library
}

\section{Michael G. Christel}

HCI Institute and

Software Engineering Inst.

Carnegie Mellon University

Pittsburgh, PA 15213 USA

mac@sei.cmu.edu
David B. Winkler

Department of Computer

Science

Carnegie Mellon Univ. Pittsburgh, PA 15213

dwinkler@cs.cmu.edu
C. Roy Taylor

Department of Computer

Science

Carnegie Mellon Univ.

Pittsburgh, PA 15213

crt@cs.cmu.edu

\begin{abstract}
Quick access to short, relevant video segments enables efficient use of a digital video library. This study evaluated an interface designed for such access, an interface that allows the user to browse a set of segments generated by a search query. Subjects' performance and attitudes were measured while they performed a fact-finding task to determine the relative effectiveness of three presentations: a text list, naively chosen visual imagery, and querybased visual imagery. Results show little difference in performance and subjective satisfaction between text lists and naively chosen imagery, but significant improvements when the images that represent video segments are based on the query that returned the segments (query-based poster frames). This result applies to the organization and representation of information in digital video libraries.
\end{abstract}

KEYWORDS multimedia, display design, evaluation, digital video library, video abstraction

\section{INTRODUCTION}

The near future will see a massive conversion of information into digital formats, a recasting of "The Information Age" as "The Digital Information Age" in which users can access almost any tidbit of data via the network - from anywhere, at any time. The resulting information will overwhelm users unless they are armed with powerful browsing and filtering support.

Digital information will include full-motion video as well as the text and single-image media found on today's Web. As digital video libraries emerge, users will need tools that handle effectively the dynamic nature of video; they will not likely invest hundreds of hours to find a single, relevant segment within a 1000 hour library. Thus future digital video libraries must support content-based indexing and retrieval of brief video segments (Brown, 1995; Taniguchi, 1995; Wactlar, 1996).

Carnegie Mellon University's Informedia Project plans to field a 1000-hour digital video library by 1998 . An initial, 45-hour library that supports content-based searching was segmented into 1600 "video clips," typically 1 to 2 minutes in length, and field-tested during the spring of 1996. Image processing, speech recognition, and natural language processing techniques were collectively applied to the video data for content extraction and temporal segmentation (Hauptmann, 1997; Smith, 1996).

\subsection{Video abstraction}

The Informedia digital video library provides users with alternate representations, video abstractions, of library materials. Users can cheaply (in terms of access, transfer, and viewing times) review a video abstraction

Human-Computer Interaction: INTERACT'97 S. Howard, J. Hammond \& G. Lindgaard (editors)

Published by Chapman \& Hall OIFIP 1997 
before deciding to incur the cost of accessing richer information layers or a complete video clip. Video abstractions used in the Informedia library include:

- Poster frame: a single "thumbnail" image representing a video clip and computed by selecting one frame from the clip and reducing it to a quarter of its original resolution, so that several representative images can be displayed simultaneously (as shown in Figures 1 and 2).

- Filmstrip: a set of images extracted from a video clip and displayed in chronological order, as shown in Figure 3. Such filmstrips have been used since the advent of digital video (Mills, 1992) and remain popular today (Taniguchi, 1995; Zhang, 1995).

- Skim: a compact representation that summarizes the original clip by concatenating its significant subsets of video and audio data.

This paper examines the impact of video abstractions, specifically poster frames, on digital video library use for a well-defined information retrieval task.

\subsection{Construction of poster frames}

Each library clip can be decomposed into camera shots, which are sets of "contiguous frames representing a continuous action in time or space" (Zhang, 1995). Informedia researchers have developed an algorithm that identifies, for each camera shot, a shot frame, the individual frame that best represents the whole shot (Smith, 1996). By default the algorithm chooses the shot's middle frame. If camera motion is detected and that motion stops within the shot, then the frame where the camera motion ends is selected. Other image processing techniques, such as those that detect and avoid low-intensity images, further refine the selection process. Thus a library clip consists of a sequence of shots and can be represented compactly by a sequence of shot frames.

Psychological research suggests that poster frames, when accompanied by text titles, allow a user to identify a video clip more quickly and accurately than do access mechanisms that present information as text only. According to theories of brain function localization and parallel cognitive processing (Guastello, 1989; Benbasat, 1993), information presented in text and pictorial form together will be processed faster than a purely textual display. Recognition may also be faster for pictures than for text (Egido, 1988; Benbasat, 1993).

However, empirical studies have not confirmed definitive advantages for iconic and pictorial representations. Specifically, studies of browsing and database navigation have found little or no performance differences between pictures and text (Egido, 1988; Benbasat, 1993). These authors conjecture that the novelty and visual appeal of pictures may slow users and offset any inherent, low-level processing advantages hypothesized for pictorial representations.

This lack of significant results may also derive from the sparse attention paid to which pictures an interface uses. Our study compared poster frames to text lists for a fact-finding task. We also examined implications of how the visual images are selected, that is, which shot frames serve as poster frames.

\section{EXPERIMENT}

\subsection{Subjects}

Thirty participants were recruited from Carnegie Mellon University students and Pennsylvania Governor's School for the Sciences high school scholarship students during the summer of 1996. Each student received $\$ 5$ for participating in this study.

Subjects were asked in an online questionnaire to rate certain experiences of the prior two weeks and six months. All subjects reported comparable experience with digital information services and little prior experience with digital video. Subjects were then randomly assigned to one of six treatment order permutations, as shown in Table 1.

\subsection{Materials}

Subjects participated in the study individually. Each used a computer with a 17-inch color monitor, headphones, and hardware support for smooth, fullmotion video playback. 


\begin{tabular}{l|c|c|c} 
& Question Set 1 & Set 2 & Set 3 \\
\hline 5 subjects & Treatment 1 (T1) & $\mathrm{T} 2$ & $\mathrm{~T} 3$ \\
\hline 5 subjects & Treatment 2 (T2) & $\mathrm{T} 1$ & $\mathrm{~T} 3$ \\
\hline 5 subjects & Treatment 3 (T3) & $\mathrm{T} 1$ & $\mathrm{~T} 2$ \\
\hline 5 subjects & $\mathrm{T} 1$ & $\mathrm{~T} 3$ & $\mathrm{~T} 2$ \\
\hline 5 subjects & $\mathrm{T} 2$ & $\mathrm{~T} 3$ & $\mathrm{~T} 1$ \\
\hline 5 subjects & $\mathrm{T} 3$ & $\mathrm{~T} 2$ & $\mathrm{~T} 1$
\end{tabular}

Table 1. Experimental design (four questions per question set)

The library for this study contained 45 hours of video from three Public Television series: "The Infinite Voyage," "Planet Earth," and "Space Age." These videos were manually segmented into 1600 clips (average length 1.7 minutes), that served as the elemental units for indexing the library. Each clip was identified with a unique seven-character text string.

Descriptive titles for each clip were added manually and speech recognition aligned each clip's text transcript with its video information (Hauptmann, 1997). This data was checked and corrected for accuracy, and the library was successfully field-tested at a local K-12 school in the spring of 1996.

To simplify the subjects' tasks, our experiment severely restricted access to the library. While recognizing the pedagogical importance of studentinitiated questions (Wallace, 1996), this study focused on the effects of different displays of query results rather than on strategies for formulating questions and searchqueries.

Following a careful analysis of the library material, we composed three question sets: "The Reign of the Dinosaurs", "Early Humans", and "Life Cycles of All Things." Pilot testing trimmed each set to four questions that:

- Were nontrivial and potentially interesting to our subjects

- Were answered completely by at least one video clip in the library

- Could be naturally rephrased into queries that return at least 12 clips from the library (the best 12 clips were presented in the study)

Each subject answered the same 12 questions ( 3 sets of 4) by examining the results of preformulated queries. The result set always contained 12 clips, as shown in Figure 1. An answer key was generated independently by other Informedia Project members. A clip in the result set was given a score of 100 if it completely answered the question, 50 if it partially answered the question, and 0 if judged not relevant.

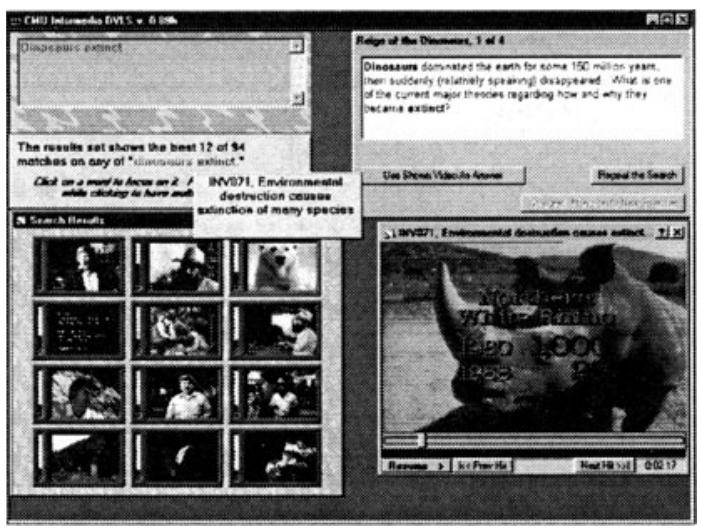

Figure 1. Screen shot (12-item result set at lower left)

\subsection{Design}

The experiment varied how video clips matching a given query were displayed in a result set: conventional text, naively chosen poster frames, and query-based poster frames. For the query illustrated in Figure 1, Figure 2 shows these three treatments. The result set was always the same size and always located at the lower left of the screen. In each treatment, moving the mouse over a result item displayed the full title text for that result item in a separate window. For example, Figure 1 shows the title window for the "polar bear" result, beginning with "INVO71."

For the text treatment, T1, each clip in the result set was represented by one line of text containing a unique ID and as many characters of its title as fit into the displayed result set area. Similar result set displays have been used by others (Brown, 1995; Hearst, 1995). A numeric query relevance score was also shown with each result line, with each result set containing 12 lines of text.

For poster frame treatments $\mathrm{T} 2$ and $\mathrm{T} 3$, the result set consisted of a $4 \times 3$ array of poster frames, one for each result. Within the border around each poster frame, a thermometer graphically indicated the query relevance score. 


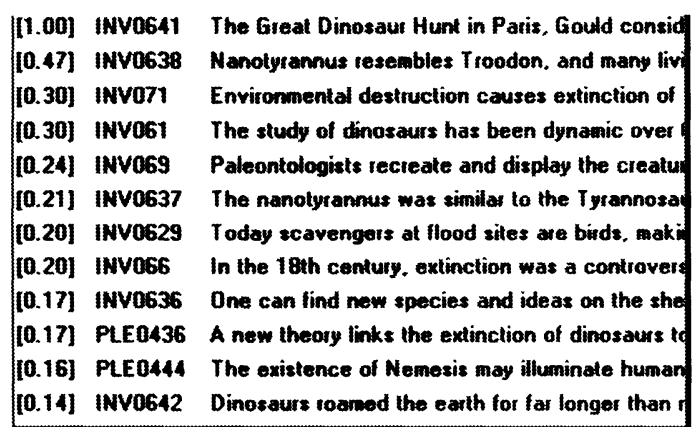

Treatment T1: Text list

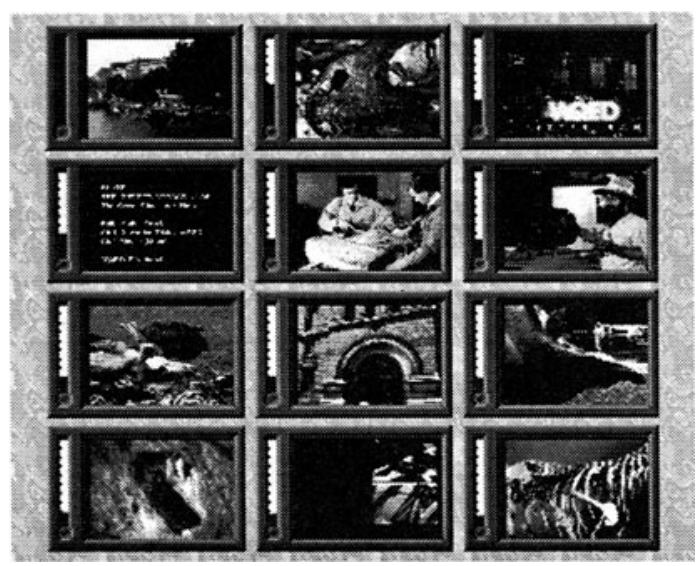

Treatment T2: Opening shot poster frames

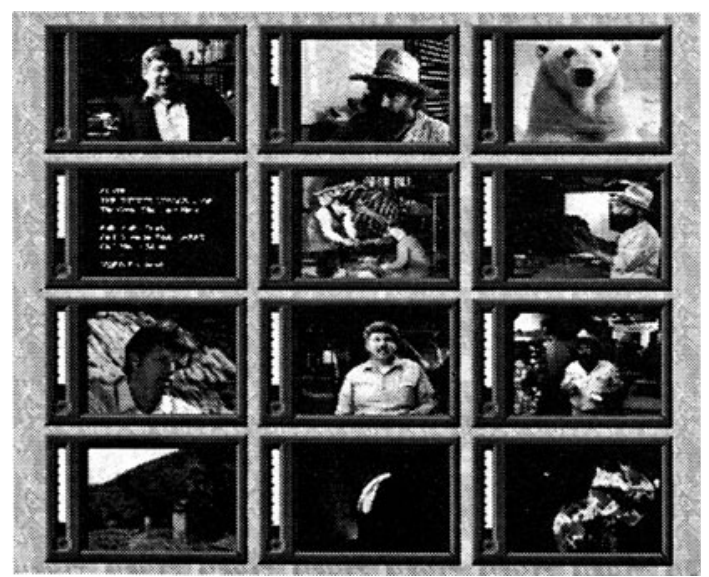

Treatment T3: Query-based poster frames

Figure 2. Results set shown in different treatments
For both treatments $\mathrm{T} 2$ and $\mathrm{T} 3$, the poster frames were computed automatically rather than manually selected. Treatment T2 used the shot frame for the first shot in the clip to represent that clip. Treatment T3 used a querybased poster frame for a clip based on the clip's set of shot frames, the time-aligned transcript, and the query text:

1. Every clip in the digital video library has a sequence of shots, each represented by a shot frame (Smith, 1996), and a transcript that is tightly aligned with the clip's video times (Hauptmann, 1997).

2. For a given query in the experiment, there are 12 clips in the result set. For each clip, words in its transcript that match the query are identified. These words each have an associated video time and a strength of match (score).

3. Each scoring time is then mapped to a single shot, and contributes to the query-matching score for that shot.

4. The shot frame for the highest scoring shot is selected as the representative image, i.e., poster frame, in the result set presentation.

This algorithm is outlined graphically in Figure 3.

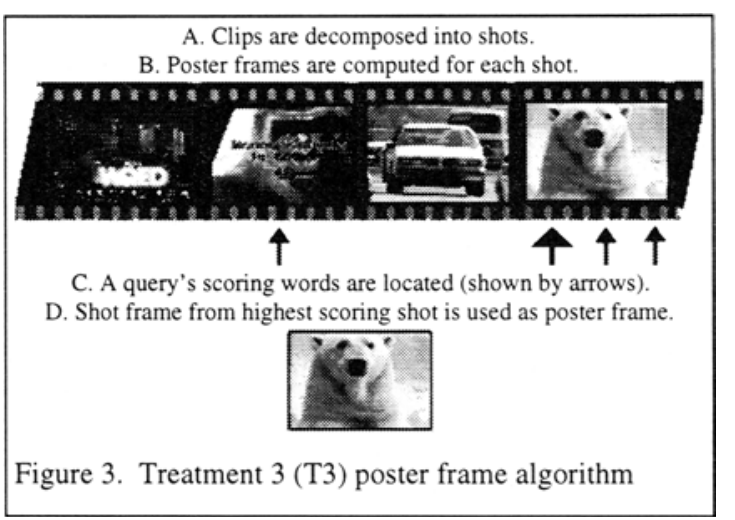

To counteract any inter-subject variability, a withinsubjects design was chosen for the experiment. Each subject experienced each treatment. All sequences of the three treatments were used to address carry-over effects, with five subjects randomly assigned to each of the six permutations of treatment order.

The dependent variables in this experiment were the grades received for each question set, the time spent to answer each question set, and the subjective satisfaction 
with the interface used for that question set. The question sets were presented in the same order to every subject. With 30 subjects, any given question set for a given treatment was seen by 10 subjects (see Table 1 ).

\subsection{Procedure}

Each subject received the same online multimedia tutorial which discussed the use of the digital library system to accomplish fact-finding tasks. Subjects answered online questionnaires concerning their backgrounds. They subsequently received instructions on the screen informing them that they would be presented with three sets of four questions each, which should be answered as quickly yet accurately as possible.

Text on the screen introduced the topic of "The Reign of the Dinosaurs." Then the first question in this set was displayed, along with the text for a related query and 12 results for that particular query (see Figure 1). The result set presentation was one of $\mathrm{T} 1, \mathrm{~T} 2$, or $\mathrm{T} 3$.

The subject browsed this result set and selected video clips to open and play until satisfied that a particular result clip was the answer to the given question. After confirming the choice, the subject was presented with the next question in the set. After all four questions in a set were answered, the subject filled in an online questionnaire concerning that set.

This procedure was then repeated two more times, for the "Early Humans" question set and "Life Cycles of All Things" question set. Each question set used a different treatment $(\mathrm{T} 1, \mathrm{~T} 2$, or $\mathrm{T} 3$ ) for their results presentations.

The Questionnaire for User Interface Satisfaction (QUIS) is a usability testing tool designed to gauge a user's subjective satisfaction with the computer interface. A subset of QUIS 5.5b-S was used for evaluation in this study, specifically all six questions concerning overall user reactions, three questions on the screen presentation $(4.1,4.2,4.3)$, and three questions on learning to use the system $(6.1,6.2,6.4)$ (QUIS, 1994). These questions were selected based on their applicability to our video library interface and the goals of this study. They formed the contents of the questionnaire filled in by each subject after each question set.

\section{RESULTS AND DISCUSSION}

No significant differences in the dependent variables were expected for treatment ordering (i.e., the random assignment to one of the six treatment permutations), nor were any found. Significant differences at the 0.05 level or better were found in a number of cases concerning the treatment variable, i.e., the manner in which the result sets were presented.

\subsection{Question set scores}

The mean question set scores for the three treatments are given in Table 2. Score differences among the treatment groups, $F(2,48)=3.103$, were significant at the $p<0.1$ level but not for $p<0.05$. We did not expect to find any significant difference for question set scores because we were using very bright high school students and college students already familiar with electronic searching and online data. These subjects were given a fairly simple task of picking a video clip from a set of 12 that contains the answer to the current query, and doing this just 4 times per question set. The trend reflected in Table 2 merits further study, however. Poster frames appear to improve scores when compared to text presentation. Differences in result set presentation may have significant effects for more difficult or more extensive fact-finding tasks.

\begin{tabular}{c|c|c}
$\begin{array}{c}\text { T1 } \\
\text { (text list) }\end{array}$ & $\begin{array}{c}\text { T2 (first shot } \\
\text { poster frame) }\end{array}$ & $\begin{array}{c}\text { T3 (query- } \\
\text { based poster } \\
\text { frame) }\end{array}$ \\
\hline 315.00 & 346.67 & 353.33
\end{tabular}

Table 2. Mean question set score per treatment (perfect score $=400$ )

\subsection{Performance time}

The mean time to complete a question set for each treatment is shown in Table 3. The treatment had significant effects on the performance time, $F(2,48)=$ $14.737, \mathrm{p}<0.001$. The query-based poster frame presentation resulted in a $36 \%$ reduction in time necessary to complete the fact-finding task.

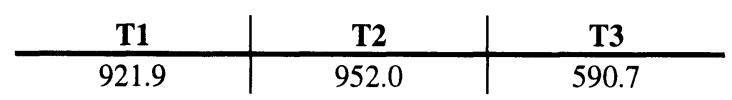

Table 3. Mean time (seconds) to complete question set 
The difference between $\mathrm{T} 2$ and $\mathrm{T} 3$ times is especially interesting, implying that simply replacing the text list with any poster frame graphical menu is not good enough. In fact, the first shot poster frame treatment had a slightly longer mean question set completion time than did the text list treatment. Only when the poster frame selection is query-based is there a significant drop in the time needed to complete the fact-finding task.

Recall that the experimental design eliminated the necessity for subjects to formulate and execute their own queries. The tasks necessary to answer the presented question were reduced to:

- Browsing the 12 video clips for candidate answers

- Playing candidate answers for appropriateness

- Selecting a video clip as the answer

Table 4 presents the mean number of video clips opened by a subject before deciding on his or her answers to a question set. Since each question set contained 4 questions, and a video clip could only be selected as an answer after it was opened, the absolute minimum number of video opens for a question set is 4 . The treatment had significant effects on the number of video opens performed in completing a question set, $F(2,48)=4.374, p<0.05$. However, this data does not explain the time difference shown in Table 3: treatments T2 and T3 both should have had much quicker task performance times based on Table 4 but Table 3 shows only $\mathrm{T} 3$ exhibiting such differences.

\begin{tabular}{c|c|c} 
T1 & T2 & T3 \\
\hline 11.63 & 9.07 & 9.83
\end{tabular}

Table 4. Mean number of video opens for question set

In support of the browsing task for finding candidate answers, subjects could move the mouse over a result set item and have a separate text box display the ID and full title string, as shown in Figure 4 for "INV071." Table 5 presents the mean number of text box titles opened by a subject in this way, referred to hence as result item browsing. The treatment had significant effects on result item browsing performed in completing a question set, $F(2,48)=8.13, \mathrm{p}<0.001$. Furthermore, the large difference between $\mathrm{T} 3$ and the other treatments helps explain the difference in time shown in Table 3.

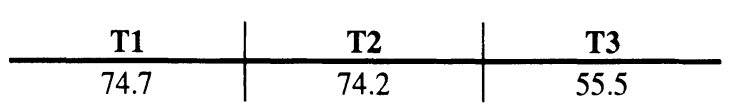

Table 5. Mean number of browsed text titles for a question set

Using the opening shot's poster frame to represent a video clip (T2) does not adequately distinguish a particular clip from others in the result set given the context of a specific query. Under T2, subjects browsed the result set as frequently as with the text lines (T1) to see the full text titles displayed for clips. When the poster frame selection is based on the query (T3), then it better identifies and distinguishes the clip, allowing users to more quickly browse the result set for candidate answers. The result is significantly less time spent browsing text titles (Table 5), good selections for candidate answers (Table 4), and an overall drop in time necessary to complete the task (Table 3).

\subsection{Subject satisfaction}

The answers to questions on screen presentation and learning to use the system produced no significant differences between the three treatment groups. The same multimedia tutorial was given to all the subjects before they began using the system. This tutorial did not bias the subjects toward a better understanding of either the text or poster frame treatments. It did serve the subjects well: the mean score for the learning questions across all subjects was 7.00 (from a 9 point scale with "9" representing the most favorable rating) and the mean score for screen presentation was 7.28.

\begin{tabular}{c|c|c} 
T1 & T2 & T3 \\
\hline 6.13 & 6.30 & 6.87
\end{tabular}

Table 6. Mean rating "terrible-wonderful" 9 pt. scale

When subjects were asked for their "overall reactions to the system as shown by this particular question set," the treatment had a significant effect on the "terrible wonderful" 9 point scale, $\mathrm{F}(2,48)=3.515$, $\mathrm{p}<0.05$. Experiences in the poster frame treatments were reported as more wonderful, and the query-based poster frame treatment receiving the most favorable rating of the three treatments. The mean scores for this question are reported in Table 6 . The other 5 questions used to 
evaluate overall subjective satisfaction revealed no significant differences.

\section{FUTURE WORK}

The results reported in the preceding section can be extended in a number of ways, including the following:

- Apply query-based techniques to other video abstractions

- Apply query-based techniques to clip boundaries

- Augment poster frames with other information visualization techniques

- Extend query-based techniques based on greater knowledge of what the user is trying to do

- Test query-based poster frame benefits for libraries containing other styles of video besides documentaries

The improvements in performance time and satisfaction brought about by the use of query-based poster frames may also be realized with other querybased video abstractions. Instead of only using a single poster frame, all scoring shots' shot frames for a particular query could be used as an abstraction for a video clip result. These frames could be displayed simultaneously in a filmstrip view, or could be presented temporally in sequence using the same screen layout as the poster frame display shown in Figure 1. Likewise, the algorithm used to define "significant" audio and video to be included in a "skim" could be strongly weighted to use the scoring data from a query. Skim videos could be dynamically adjustable with parameters both for collapsed playback rate (e.g., 0 to $50 \%$ of original playback time) and the amount of influence of a query in composing the skim. Empirical studies will have to be conducted to determine the benefits of querybased modifications to a video abstraction when that abstraction has a temporal component.

Perhaps the query-based modifications should be extended to the clip boundaries as well for material in a digital video library. A physical segmentation of the video library into clips imposes a fixed segmentation on the video data. These clips are separated from their original context and may not meet the future needs of the library user (Davis, 1994). A more flexible alternative is to logically segment the library into clips but keep the video data intact in its original context, as is done with the Informedia library (Wactlar, 1996).
Tests can be run to determine the effects of dynamically modifying clip boundaries based on the query or other user action which retrieves that clip.

A number of other browsing mechanisms could be added to the query results display for the video library. To enable better filtering and browsing, the features deemed important by the user should be emphasized and made visible. For example, if the distribution behavior of query terms within a video clip is important to a user, TileBars could be used in presenting the query results (Hearst, 1995). Further work must be done to explore the effects of using browsing mechanisms like TileBars in combination with video abstractions when accessing digital video libraries.

To determine which browsing mechanisms are appropriate to a user's needs, the user's identity and primary tasks must be better understood. Query-based poster frames produced improvements when the task centered about the query results and browsing the 12 video clips for candidate answers. For tasks not as dependent on queries, other browsing mechanisms may become critical or the query-based poster frame selection may need to be refined. For example, digital libraries for the sciences in support of inquiry-based learning should allow students to investigate driving questions by "asking and refining questions, making plans, designing experiments, debating ideas, collecting and analyzing information and data, drawing conclusions and communicating their ideas and findings to others" (Wallace, 1996). The use of query-based poster frames would likely benefit the analysis task when viewing a collection from a query. If given a larger context, however, such as inquiry-based learning, then video abstractions could be further refined for added benefit within that domain.

The library used in the experiment reported here contained all documentaries. The results should be verified across other classes of video, such as news footage which is often the focus of other research groups (Brown, 1995; Zhang, 1995; Hauptmann, 1997).

\section{CONCLUSIONS}

Full content retrieval in a video library can be a difficult and time-consuming process, as evidenced by anyone trying to find a clip of interest in a videotape or, worse, from a stack of videotapes. In order to improve 
access to relevant information, a digital library can be logically partitioned into clips and can provide alternate abstractions of video content. These abstractions can effectively represent a clip and be viewed in less time than the full clip. The experiment reported here shows that a single, representative image for a clip - when based on query criteria - improves performance time and subjective satisfaction.

The future promises to deliver increasingly more powerful computer processing and greater bandwidth. Digital video will no longer be relegated to the multimedia research labs or a few precious hours on a home machine, but will become as commonplace as text and graphics. The difficulties in quickly accessing a video clip of interest will remain, however, underscoring the importance of improving video abstractions.

\section{ACKNOWLEDGMENTS}

This material is based on work supported by the National Science Foundation, DARPA, and NASA under NSF Cooperative Agreement No. IRI-9411299. We thank Howard Wactlar for his project leadership, Michael Smith for his work with video abstractions, and Bryan Maher, Ricky Houghton, Craig Marcus, King Sun Wai, Don Marinelli, Michael Wagner, and the rest of the Informedia Project team for their efforts in preparing the library for experimentation.

\section{REFERENCES}

Benbasat, I. And Todd, P. (1993) An experimental investigation of interface design alternatives: icon vs. text and direct manipulation vs. menus. Int. Journal of Man-Machine Studies, 38, 369-402.

Brown, M.G., Foote, J.T., Jones, G.J.F., Jones, K.S., and Young, S.J. (1995) Automatic Content-Based Retrieval of Broadcast News. In Proceedings of the ACM Multimedia Conference, 35-43.

Davis, M. (1994) Knowledge Representation for Video. In Proceedings of AAAI '94, 120-127.

Egido, C. and Patterson, J. (1988) Pictures and Category Labels as Navigational Aids for Catalog Browsing. In Proceedings of the ACM CHI'88 Conference on Human Factors in Computing Systems, 127-132.
Guastello, S.J., Traut, M., and Korienek, G. (1989) Verbal versus pictorial representations of objects in a human-computer interface. Int. Journal of ManMachine Studies, 31, 99-120.

Hauptmann, A.G., and Witbrock, M.J. (1997) Informedia: News-on-Demand - Multimedia Information Acquisition and Retrieval. To appear in Intelligent Multimedia Information Retrieval (ed. M. Maybury), MIT Press, Menlo Park, CA.

Hearst, M.A. (1995) TileBars: Visualization of Term Distribution Information in Full Text Information Access. In Proceedings of the ACM CHI'95 Conf. on Human Factors in Computing Systems, 59-66.

Mills, M., Cohen, J., and Wong, Y.Y. (1992) A Magnifier Tool for Video Data. In Proceedings of the ACM CHI'92 Conference on Human Factors in Computing Systems, 93-98.

QUIS 5.5b. (1994) University of Maryland at College Park. Available through http://www.lap.umd.edu/ QUISFolder/quisHome.html.

Smith, M. and Kanade, T. (1996) Video Skimming for Quick Browsing Based on Audio and Image Characterization. Carnegie Mellon University, School of CS Technical Report CMU-CS-95-186R, Pittsburgh, PA.

Taniguchi, Y., Akutsu, A., Tonomura, Y., and Hamada, H. (1995) An Intuitive and Efficient Access Interface to Real-Time Incoming Video Based on Automatic Indexing. In Proceedings of the ACM Multimedia Conference, 25-33.

Wactlar, H.D., Kanade, T., Smith, M.A., and Stevens, S.M. (1996) Intelligent Access to Digital Video: Informedia Project. Computer, 29(5), 46-52.

Wallace, R., Krajcik, J., and Soloway, E. (1996) Digital Libraries in the Science Classroom: An Opportunity for Inquiry. D-Lib Magazine. URL http:// www.dlib.org/dlib/september96/09contents.html.

Zhang, H.J., Smoliar, S.W., Wu, J.H., Low, C.Y., and Kankanhalli, A. (1995) A Video Database System for Digital Libraries. In Digital Libraries: Current Issues (Digital Libraries Workshop DL '94, Newark, NJ, May 1994, Selected Papers) (eds. N.R. Adam, B.K. Bhargava, and Y. Yesha), Springer, Berlin. 